\title{
Jumping Breast Phenomenon Following Subcutaneous Mastectomy: First Description and Grading of a Well-Known Breast Deformity
}

\author{
Sherko Kümmel ${ }^{\mathrm{a}} \quad$ Aylin Kümmel $^{\mathrm{a}}$ Friederike Hagemann $^{\mathrm{a}} \quad$ Anna Rüland $^{\mathrm{a}}$

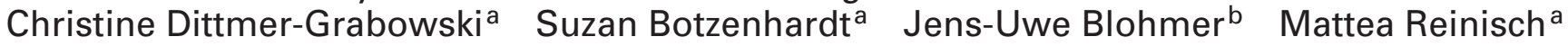 \\ ${ }^{a}$ Breast Unit, Evangelische Huyssens-Stiftung, Kliniken Essen-Mitte, Essen, Germany; \\ ${ }^{b}$ Department of Obstetrics and Gynaecology, Breast Unit, Charité University Medicine, Campus Mitte and Benjamin Franklin, \\ Berlin, Germany
}

\section{Keywords}

Breast distortion after subcutaneous mastectomy . Implants · Reconstruction - Breast operation ·

Breast cancer

\section{Summary}

Introduction: One of the goals of breast cancer surgery is to preserve the breast. However, where this is not possible, various breast reconstructive procedures are available. The most common procedure is the immediate insertion of a subpectoral implant after removing the breast tissue. A significant challenge involving subpectoral implants is the deformation of the breast upon contraction of the pectoral muscle causing elevation of the breast and development of wrinkles or ripples in the caudal and cranial quadrants (jumping breast), especially in slim patients with a thin subcutaneous fat layer. Methods: While the jumping breast phenomenon after augmentation is well-known in cosmetic breast surgery, it has never been systematically described. In oncologic breast surgery, this deformity, which at times can be quite severe, has been ignored thus far. Results and Conclusion: In this paper, we present an initial distinction of grades for the so-called jumping breast, show examples for the different grades of severity of breast deformity in response to tensing of the pectoral muscle, and further describe different primary and secondary procedures for the prevention of the jumping breast phenomenon. By means of a 2-stage procedure, we can prevent this complication and reduce the risk of breast deformity after implant-based breast reconstruction.

(c) 2018 S. Karger GmbH, Freiburg

\section{Introduction}

In recent decades, there has been continuous improvement in the overall survival of patients with breast cancer due to advances in systemic treatment. After it was recognized that radical surgery does not reduce mortality, modified radical mastectomy was introduced, followed by breast-conserving treatment $[1,2]$. However, in approximately one-third of breast cancer patients, breast-conserving treatment is not performed due to multicentricity, evidence of a BRCA1/2 mutation, large tumor size, or presence of a large-size precancerous stage (ductal carcinoma in situ) leading to an unfavorable breast-tumor ratio. Under these conditions, physicians and patients often choose an ablative procedure. Given the increasing improvement in treatment and the reduction in mortality, the prior primary focus on purely oncologically-oriented breast cancer surgery has yielded in part to the achievement of also esthetically satisfying results. If the patient desires the restoration of her original appearance, a direct reconstruction should be carried out [3]. In general, subcutaneous mastectomy with preservation of the nipple and areola yields the most satisfactory cosmetic result, without oncologic risks to the patient.

A distinction is made between autologous and heterologous reconstruction. Both methods have their advantages and disadvantages. There are no randomized studies comparing the 2 forms of reconstruction with respect to cosmetic results, patient satisfaction, rate of complications, and oncologic reliability [4]. In the majority of cases, direct reconstruction is done by means of a heterologous implant which can be placed either prepectorally or subpectorally. Possible risks and complications of implant-based breast reconstruction are the emergence of capsule fibrosis $[5,6]$, dislocation of the implant, a foreign body-like feeling in the breast, numbness [7-10], re-operation, and adhesion of the breast muscle to the

\section{KARGER}

(c) 2018 S. Karger GmbH, Freiburg 
overlying skin; via tension in the pectoral muscle, the latter can lead to the unnatural breast deformity known as 'jumping breast'.

To our knowledge, the jumping breast phenomenon has not been described in detail in any journal of oncologic surgery thus far. The existence of this breast deformity is well known; synonyms include 'breast animation', 'breast (shape) distortion', 'unnatural movement with chest muscle contraction', or 'animation deformities'. While inconsistent categorization of this complication as 'mild to severe' can be found in the literature, the variables underlying these categories have never been described in detail and characterization remains vague [11]. Thus, a comparison of incidences is not possible. In the present paper, the jumping breast phenomenon following subcutaneous mastectomy is analyzed to establish objectifiable criteria to facilitate the comparison of incidence and severity in the future. Furthermore, we describe the procedure followed at our institution to avoid the occurrence of jumping breast and give a brief summary of various surgical methods to correct this phenomenon.

\section{Patients and Methods}

\section{Jumping Breast Phenomenon}

The expressions 'breast animation', 'breast (shape) distortion', 'unnatural movement with chest muscle contraction', 'animation deformities', or 'hyperanimation' describe for the most part breast deformities that occur after esthetic breast augmentation $[7-10,12,13]$. In contrast to esthetic augmentation, in the case of subcutaneous or skin-sparing mastectomy due to breast cancer, the glandular tissue is completely removed leaving the overlying skin and varying amounts of subcutaneous fat tissue, and possibly the nipple-areola complex in the case of a purely subcutaneous mastectomy. In heterologous breast reconstruction, the implant can be inserted in different ways: either primarily prepectoral or primarily subpectoral. Both methods have their benefits and drawbacks. Disadvantages of a prepectoral implant that have been described in the literature are a secondary bottoming out or visible wrinkling of the implant. What has to be further taken into consideration in the setting of mastectomy is that only in the rarest of cases a fat layer of more than $2 \mathrm{~cm}$ can remain. In particular, in slim patients with a thin subcutaneous fat layer and a pinch test of $<1-2 \mathrm{~cm}$, a subpectoral implant for reconstruction after mastectomy is advantageous for achieving a good cosmetic result since otherwise the contour of the implant is frequently visible.

However, primary subpectoral implants carry the risk that during the healing process extensive adhesions between the pectoral muscle and the overlying skin can develop. If this occurs, the skin moves in sync with the pectoral muscle upon contraction, resulting in an unnatural cranial movement of the reconstructed breast with formation of wrinkles or ripples in the caudal and/or cranial area and frequently an additional distortion of the nipple-areola complex. The breast 'jumps' cranially ('jumping breast'). This can strongly affect the cosmetic result since, depending on the severity of the condition and the patient's constitution, this deformity can lead to an asymmetry of the breasts and may be visible in the chest area even when the patient is fully dressed.

\section{Results}

To establish a classification for the grade of severity of the jumping breast phenomenon, an objective system of evaluation with the help of a 3-point scale was set up (table 1). Drawing on the classification of the degree of severity by Baker et al. [11], the objectively determinable movement of the breast upon muscle contraction in the caudal and cranial quadrants is evaluated while also taking patient satisfaction into account.

Figures 1-4 give examples for jumping breast grade $1-3$. The photos show the breast after reconstruction in a resting position (A) and upon tensing of the pectoral muscles through the patient pressing her hands together (B). Figure 1 clearly shows that contraction of the pectoral muscles leads to rippling in the lower quadrants with contralateral asymmetry and elevation of the nippleareola complex. The upper quadrant is shorter as a result of the elevation of the nipple-areola complex but there is no visible rippling or change in the cosmetic result in the upper quadrants. Both nipple-areola complexes are raised to the same extent during contraction of the pectoral muscles. As the horizontal bar clearly shows, the elevation of the nipple-areola complex is about $50 \%$ of its size. The marked area in the right breast shows the shortening of the upper quadrants. Rippling in the area of the caudal quadrants upon contraction is particularly visible on the left side.

Figure 2 shows a grade 2 jumping breast. Contraction of the pectoral muscles produces clear wrinkling with elevation of the nipple-areola complex and shortening of the upper quadrant.

Figure 3 clearly shows rippling in the area of the upper and lower quadrants upon contraction of the pectoral muscles (fig. 3B). The nipple-areola complex does not significantly change position.

Table 1. Grading of the jumping breast phenomenon (based on bilateral evaluation)

\begin{tabular}{ll}
\hline Grade & Features \\
\hline 1 & $\begin{array}{l}\text { wrinkles or rippling in the breast compared to the other side, } \\
\text { without an influence on the contour of the upper quadrants, } \\
\text { possibly with a shift in the nipple-areola complex (caudal } \\
\text { jumping breast) }\end{array}$ \\
\hline 2 & $\begin{array}{l}\text { pronounced formation of wrinkles or rippling in the skin of } \\
\text { the upper-inner quadrants, with a possible shift in the nipple- } \\
\text { areola complex (cranial jumping breast) }\end{array}$ \\
\hline 3 & $\begin{array}{l}\text { combined cranial and caudal jumping breast, with a possible } \\
\text { shift in the nipple-areola complex }\end{array}$
\end{tabular}

Note: If the breast deformity is causing discomfort, the grade is at least 2 .
Fig. 1. A, B Image of jumping breast grade 1 , right side.

\section{A}

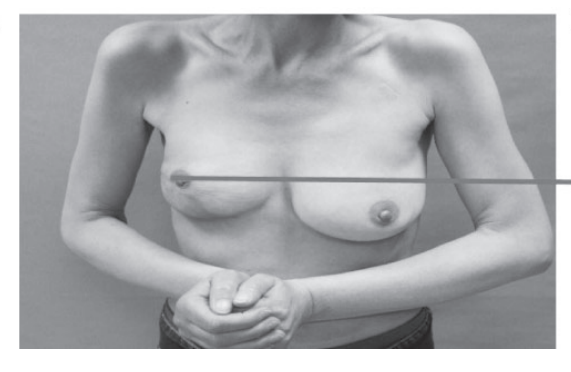

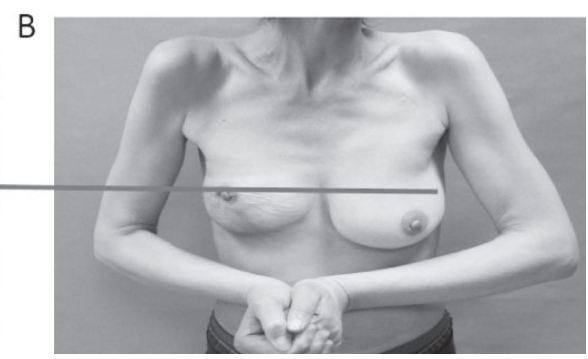


Fig. 2. A, B Example of jumping breast grade 2 after bilateral subcutaneous mastectomy.

Fig. 3. A, B Image of bilateral grade 3 jumping breast.

Fig. 4. A-D Example of a combined grade 3 (right) and grade 2 (left) jumping breast.

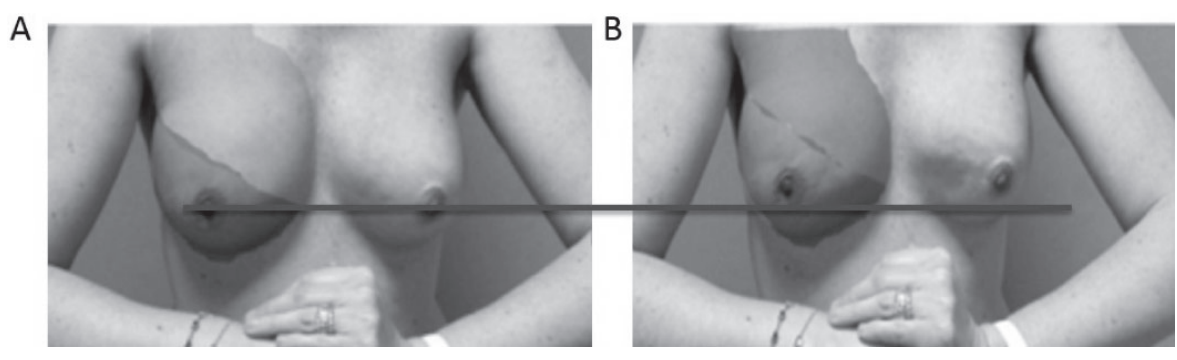

A

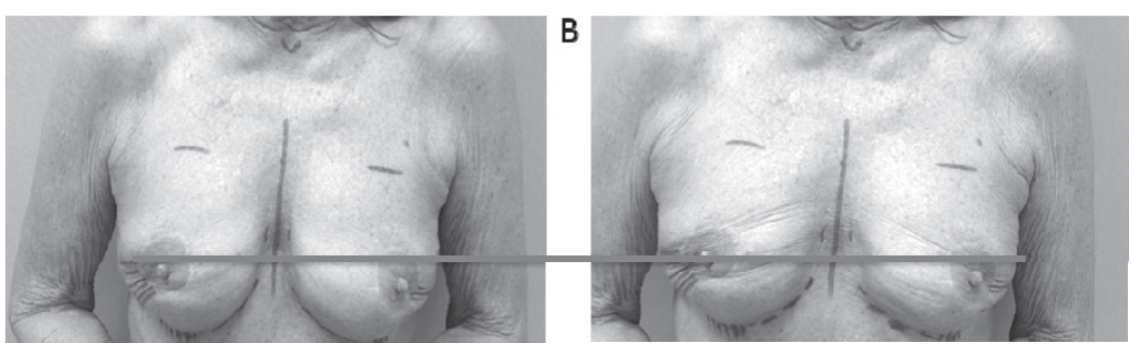

A

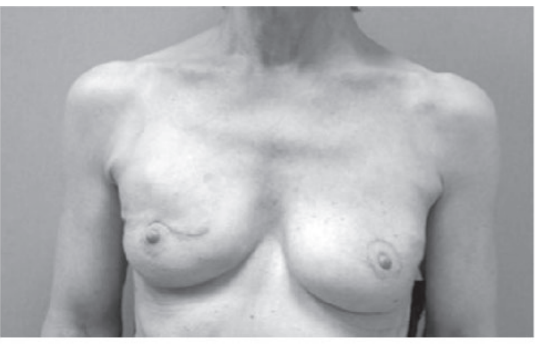

B

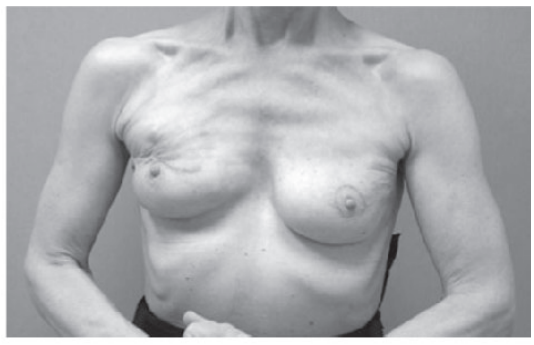

C

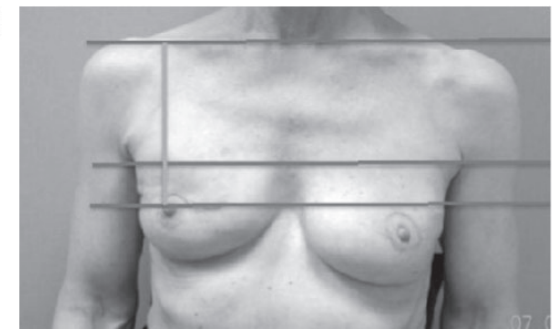

D

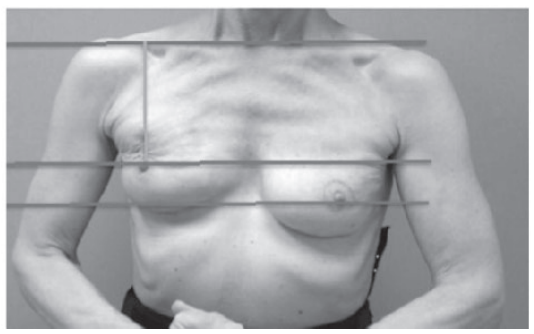

Figure 4 shows an extreme grade 3 jumping breast on the right. The difference compared to grade 2 is clear - there is no change in contour in the caudal quadrant but strong rippling in the lateral upper quadrant with elevation of the nipple-areola complex. In this case, the elevation of the nipple-areola complex is over the entire upper quadrant (horizontal bar) and the clavicular distance to the nipple-areola complex is significantly reduced (vertical line), as is shown by the lines in the lower part of figure $4(\mathrm{C} / \mathrm{D})$.

\section{Discussion}

Breast deformity following subcutaneous mastectomy and reconstruction of the breast by means of subpectoral implants is a well-known problem. It results from adhesion of the pectoral muscle to the overlying skin with contraction of the muscle leading to a cranial shift of the breast of varying degree. Various isolated solutions for this problem have been described, but so far a scientific investigation of the so-called jumping breast phenomenon, including a definition of grades, has been lacking.

In the present paper, we introduce for the first time a grade classification for the jumping breast phenomenon to facilitate the comparison of this breast deformity in the future; we also show examples of cases to demonstrate the grades and briefly present solutions for the problem.

The best therapy for jumping breast is primary prevention. By means of a 2-stage procedure, as has become standard in our clinic, we prevent the emergence of this complication. First, the mammary gland is completely removed (usually with retention of the nipple-areola complex) by making an incision in the inframammary fold, followed by prepectoral implant insertion. Physiologically, a smooth thin capsule containing little collagen forms around the implant. Since the implant lies between the muscle and the skin, no adhesion occurs between the 2 layers. As a result, there is no visible change in the position or form of the breast upon contraction of the pectoral muscles. If the result is cosmetically accept- 
able, the second step is optional. A second intervention may be indicated, e.g. in cases of macromastia where a reduction of the overlying skin is indicated, or where there is a thin subcutaneous fat layer with a recognizable or palpable implant contour, or in cases of secondary ptosis. In such cases, at the earliest 3 months after the first implantation, a subpectoral transfer of the implant is carried out without resection of the capsule. The capsule now functions as a kind of 'slide bearing' between the muscle and the skin so that contractions of the muscle are not transferred to the skin. In addition, the physiologically formed capsule provides protection for the lower end of the implant, which otherwise often has to be installed by insertion of matrices or mesh.

Cosmetic adjustments such as reduction of the overlying skin by means of a skin lift or volume correction of the implant can be carried out with minimal complications during the second intervention in order to change the level of the implant. In the case of macromastia and a necessary reduction of the overlying skin, the 2-stage intervention reduces the risk of necrosis of the nipple-areola complex [14]. Due to neoangiogenesis in the area of the nippleareola complex after subcutaneous mastectomy, sufficient oxygenation continues to be provided in the case of a second surgery to change the location of the nipple-areola complex or reduce overlying skin.

Cosmetically less satisfactory is the outcome of solutions where by means of denervation or division of the pectoral muscle an attempt is made to reduce the extent of the jumping breast affect. Here, it is possible to carry out a partial or complete transection of the pectoral muscle in the area of the axilla or even sever the nervus pectoralis lateralis or medialis. There is little data in the literature on these treatment options or descriptions of success rates. Difficulties arise in terms of the clear identification of the nerve branch in situ due to variations in anatomy [15], risk of iatrogenic denervation of a false nerve branch, or incomplete denervation of nerve structures serving the pectoral muscle due to various collateral effects, with the result that the jumping breast remains. Problems also frequently arise due to scars and tissue structures from prior operations.

There are no studies comparing the above methods or their success rates. Our own clinical experience shows an unsatisfactory success rate and repeated reoperations after denervation of the pectoral muscle. With these re-operations, the patient runs the risk of further adhesions, scar extrusions that limit movement, and a poor cosmetic result, as well as the general risks of infection. The insertion of an acellular dermal matrix (ADM) has been discussed in the literature [16] in conjunction with subcutaneous mastectomy and prepectoral implants, with the description of 22 satisfactory cosmetic results. However, this paper already discussed the drawbacks of subcutaneous implantation and highlighted the need for alternative solutions. Another work discussing prepectoral versus subpectoral breast reconstruction is by Nahabedian et al. [17]. In this retrospective review, all patients received ADM. The authors reported a generally higher rate of adverse events in the group receiving partial subpectoral reconstruction versus prepectoral reconstruction ( 22 vs. $20.5 \%$ ). In this work, no information is given on breast de- formity. Further, in a meta-analysis [12] of 14 studies comparing prepectoral versus subpectoral implant-based breast reconstruction, no hyperanimation was found in the cohort of prepectoral breast reconstruction. The authors concluded that due to the comparable adverse events profile, both techniques are feasible options for breast reconstruction in eligible patients. The generally welltolerated prepectoral-based breast reconstruction without the risk of a jumping breast or other kinds of breast deformity is further supported by the experience with 350 cases reported by Sigalove et al. [18]. The authors did not experience breast animation with prepectoral-based breast reconstruction based on the use of ADM, autologous fat cells transfer to augment the soft tissue coverage of the implant, and cohesive gel implants to recreate the breast.

Our experience shows that in the majority of cases there is no need for insertion of an ADM and that it does not fully overcome the risk of deformities due to formation of edges or wrinkling. On the contrary, it can result in painful adhesions that can also lead to breast deformity. In thin patients, the suture can be palpable subcutaneously, and further diagnostics may be needed to differentiate this from local recurrence of breast cancer. Furthermore, in our experience, the risk of capsule fibrosis remains, and intolerances with respect to ADMs have been described in the literature $[19,20]$.

In contrast, in our experience, the reverse change of position from subpectoral to prepectoral has a high success rate in a select collective. However, supporting data is currently limited. Preconditions for the procedure are the preservation of an adequate subcutaneous skin overlay with a pinch test of $>1-2 \mathrm{~cm}$, as well as an inconspicuous breast contour in the resting breast without contraction of the pectoral muscle.

The triple plane technique [9] is employed in cosmetic breast augmentation and is also used for the primary prevention of breast deformity after augmentation. Here, the pectoral muscle is cut horizontally to the sternal base on the level of the nipple-areola complex. The authors describe retention of muscle strength with a reduction in breast deformity. In $67 \%$ of the cases after triple plane surgery there was no breast deformity, in $29.77 \%$ there was slight breast deformity. The classification used by Spear et al. [11] describes the extent of breast deformity based on subjective judgment (mild to severe) and on the visibility of the implant (whether it is recognizable pre- or subpectorally). However, in cases of prepectoral mastectomy based on an oncologic indication, this procedure and classification are inadequate as they ignore the location of the breast deformity. In addition, it seems inadvisable to perform a horizontal incision of the pectoral muscle at the level of the nippleareola complex after removal of the breast tissue.

Despite being widespread and widely accepted, the jumping breast phenomenon after subcutaneous mastectomy in patients with breast cancer has thus far not been scientifically graded [12]. As a result of this lack of a standardized means to describe its severity, the jumping breast phenomenon can neither be compared intra-institutionally nor inter-institutionally. The lack of uniform descriptions in the literature of changes in the breast after augmentation makes comparisons of the severity between institutions difficult as well as having negative consequences for the patients. 
The present paper describes a grade classification for the jumping breast phenomenon that takes into account both objective criteria and the subjective perception of the patient. This grading system was developed on the basis of discussions with certified breast surgeons from different institutions. Reproducibility of the results between different physicians was also taken into consideration. Although the jumping breast represents no risk oncologically and causes no pain, grades 2 and 3 in particular can result in a significant reduction in the quality of life of the affected patients. In addition to the oncologic success, the surgical cosmetic result with high patient satisfaction after breast reconstruction following breast cancer is a prime concern. Breast deformities should be avoided. In addition to the retention of the overlying skin and the choice of where the incision is going to be made, surgical planning has to take into account the risk of scar formation, adhesions, and fatty tissue necrosis. The goal of the description of the jumping breast phenomenon with the establishment of an objective grading system was to draw the attention of the surgeons to this condition. Subsequent papers will describe the applicability of this grading classification and the rate of success of the surgical method introduced at our institution, as well as the patients' rate of satisfaction.

\section{Disclosure Statement}

The authors declare no competing interest.

\section{References}

1 Fisher B, Anderson S, Bryant J, Margolese RG, Deutsch M, Fisher ER, Jeong JH, Wolmark N: Twenty-year follow-up of a randomized trial comparing total mastectomy, lumpectomy, and lumpectomy plus irradiation for the treatment of invasive breast cancer. N Engl J Med 2002;347:1233-1241.

2 Al-Ghazal SK, Fallowfield L, Blamey RW: Comparison of psychological aspects and patient satisfaction following breast conserving surgery, simple mastectomy and breast reconstruction. Eur J Cancer 2000;36:19381943.

$\checkmark 3$ Robert Koch-Institut, Gesellschaft der epidemiologischen Krebsregister in Deutschland e.V.: Krebs in Deutschland 2009/2010 2013, ed 9. 2013.

4 Shea-Budgell M, Quan ML, Mehling B, Temple-Oberle C: Breast reconstruction following prophylactic or therapeutic mastectomy for breast cancer: recommendations from an evidence-based provincial guideline. Plast Surg (Oakv) 2014;22:103-111.

5 Headon H, Kasem A, Mokbel K: Capsular contracture after breast augmentation : an update for clinical practice. Arch Plast Surg 2015;42:532-543.

6 Handel N, Cordray T, Gutierrez J, Jensen JA: A longterm study of outcomes, complications, and patient satisfaction with breast implants. Plast Reconstr Surg 2006;117:757-772.

7 Spear SL, Schwartz J, Dayan JH, Clemens MW: Outcome assessment of breast distortion following submuscular breast augmentation. Aesthetic Plast Surg 2009;33:44-48.
8 Pelle-Ceravolo M, Del Vescovo A, Bertozzi E, Molinari P: A technique to decrease breast shape deformity during muscle contraction in submuscular augmentation mammaplasty. Aesthetic Plast Surg 2004;28288-28294.

9 Bracaglia R, Tambasco D, Gentileschi S, D'Ettorre M: Triple-plane technique for breast augmentation: solving animation deformities. Aesthetic Plast Surg 2013; 37:715-718.

10 Hammond DC, Schmitt WP, O’Connor EA: Treatment of breast animation deformity in implant-based reconstruction with pocket change to the subcutaneous position. Plast Reconstr Surg 2015;135:1540-1544.

11 Spear S, Baker JJ: Classification of capsular contracture after prosthetic breast reconstruction. Plast Reconstr Surg 1995;96:1119-1123.

12 Chatterjee A, Nahabedian MY, Gabriel A, Macarios D, Parekh M, Wang F, Griffin L, Sigalove S: Early assessment of post-surgical outcomes with pre-pectoral breast reconstruction: a literature review and metaanalysis. J Surg Oncol 2018;117:1119-1130.

13 Chatterjee A, Nahabedian MY, Gabriel A, Sigalove S, Parekh M, Wang F, Griffin L, Macarios D: Early assessment of post-surgical outcomes with pre-pectoral breast reconstruction: a literature review and metaanalysis. Plast Reconstr Surg Glob Open 2017;5 (suppl):23.

14 Arens-Landwehr A, Klasmeyer K, Liebau J: Vermeidung von Komplikationen bei der Mammareduktionsplastik. J Ästhetische Chir 2011;4:20-27.
5 Prakash KG, Saniya K: Anatomical study of pectora nerves and its implications in surgery. J Clin Diagnostic Res 2014;8:AC01-5.

16 Reitsamer R, Peintinger F: Prepectoral implant placement and complete coverage with porcine acellular dermal matrix: a new technique for direct-to-implant breast reconstruction after nipple-sparing mastectomy. J Plast Reconstr Aesthet Surg 2015;68:162-167.

17 Nahabedian MY, Cocilovo C: Two-stage prosthetic breast reconstruction. Plast Reconstr Surg 2017;140: 22S-30S.

18 Sigalove S, Maxwell GP, Sigalove NM, Storm-Dickerson TL, Pope N, Rice J, Gabriel A: Prepectoral implant-based breast reconstruction: rationale, indications, and preliminary results. Plast Reconstr Surg 2017;139:287-294.

19 Kim JYS, Davila AA, Persing S, Connor CM, Jovanovic B, Khan SA, Fine N, Rawlani V: A meta-analysis of human acellular dermis and submuscular tissue expander breast reconstruction. Plast Reconstr Surg 2012;129:28-41.

20 Winocour S, Martinez-Jorge J, Habermann E, Thomsen K, Lemaine V: Early surgical site infection following tissue expander breast reconstruction with or without acellular dermal matrix: national benchmarking using National Surgical Quality Improvement Program. Arch Plast Surg 2015;42:194-200. 